\title{
Fiducial markers coupled with 3D PET/CT offer more accurate radiation treatment delivery for locally advanced esophageal cancer
}

\section{(ㄷ)(1) $(2) \Theta$}

\section{Authors}

Jasmine A. Oliver ${ }^{1,2}$, Puja Venkat ${ }^{1}$, Jessica M. Frakes ${ }^{1}$, Jason Klapman ${ }^{3}$, Cynthia Harris ${ }^{3}$, Jaime Montilla-Soler ${ }^{4}$, Gautamy C. Dhadham ${ }^{3}$, Baderaldeen A. Altazi ${ }^{1,2}$, Geoffrey G. Zhang ${ }^{1,2}$, Eduardo G. Moros ${ }^{1,2}$, Ravi Shridhar ${ }^{5}$, Sarah E. Hoffe ${ }^{1}$, Kujtim Latifi ${ }^{1,2}$

Institutions

1 H. Lee Moffitt Cancer Center and Research Institute, Department of Radiation Oncology, Tampa, FL, USA

2 University of South Florida, Department of Physics, Tampa, FL, USA

3 H. Lee Moffitt Cancer Center and Research Institute, Gastrointestinal Tumor Program, Division of Endoscopic Oncology, Tampa, FL, USA

4 H. Lee Moffitt Cancer Center and Research Institute, Department of Diagnostic Imaging, Tampa, FL, USA

5 Florida Hospital Cancer Institute, Orlando, FL, USA

submitted 8.9.2016

accepted after revision 1.2 .2017

Bibliography

DOI https://doi.org/10.1055/s-0043-104861 |

Endoscopy International Open 2017; 05: E496-E504

(c) Georg Thieme Verlag KG Stuttgart · New York

ISSN 2364-3722

Corresponding author

Kujtim Latifi, PhD, Department of Radiation Oncology, Moffitt Cancer Center (RAD ONC), 12902 Magnolia Drive, Tampa, FL 33612, USA

Fax: +1-813-449-8978

Kujtim.Latifi@Moffitt.org

\section{ABSTRACT}

Background and aims The role of three-dimensional positron emission tomography/computed tomography (3D $\mathrm{PET} / \mathrm{CT}$ ) in esophageal tumors that move with respiration and have potential for significant mucosal inflammation is unclear. The aim of this study was to determine the correlation between gross tumor volumes derived from $3 \mathrm{D}$ PET/CT and endoscopically placed fiducial markers.

Methods This was a retrospective, IRB approved analysis of 40 patients with esophageal cancer with fiducials implanted and PET/CT. The centroid of each fiducial was identified on PET/CT images. Distance between tumor volume and fiducials was measured using axial slices. Image features were extracted and tested for pathologic response predictability.

Results The median adaptively calculated threshold value of the standardized uptake value (SUV) to define the metabolic tumor volume (MTV) border was 2.50, which corresponded to a median $23 \%$ of the maximum SUV. The median distance between the inferior fiducial centroid and MTV was $-0.60 \mathrm{~cm}(-3.9$ to $2.7 \mathrm{~cm})$. The median distance between the superior fiducial centroid and MTV was $1.25 \mathrm{~cm}$ $(-4.2$ to $6.9 \mathrm{~cm})$. There was no correlation between MTVto-fiducial distances greater than $2 \mathrm{~cm}$ and the gastroenterologist who performed the fiducial implantation. Eccentricity demonstrated statistically significant correlations with pathologic response.

Conclusions There was a stronger correlation between inferior fiducial location and MTV border compared to the superior extent. The etiology of the discordance superiorly is unclear, potentially representing benign secondary esophagitis, presence of malignant nodes, inflammation caused by technical aspects of the fiducial placement itself, or potential submucosal disease. Given the concordance inferiorly and the ability to more precisely set up the patient with daily image guidance matching to fiducials, it may be possible to minimize the planning tumor volume (PTV) margin in select patients, thereby, limiting dose to normal structures.

\section{Introduction}

The 5 year survival rate for esophageal cancer patients is $18 \%$ and there were approximately 17000 estimated new esophageal cancer cases and nearly 16000 estimated deaths in 2016 [1]. This is perpetuated because most patients with esophageal cancer present with locally advanced or metastatic disease [2]. To improve outcomes, neoadjuvant therapy is recommended for patients with locally advanced disease before surgery [3]. At present, data on individual sensitivity to chemotherapy and radiation therapy are lacking, thus patients are advised to un- 
dergo standard of care chemoradiation based on their clinical, rather than molecular, factors. Clinicians rely on radiographic indicators to assess response but, in the absence of progression at restaging, patients proceed to esophageal resection; the quality of life implications and medical cost of this are profound if patients have a pathologic complete response and yet have undergone removal of their esophagus. Conversely, if patients are found at the time of surgery to have had no response, their outcomes are no better than if they went directly to surgery upfront [4]. In fact, in the case of the pathologic non-responder, there is also the consideration of the potential acute neoadjuvant toxicity incurred for no demonstrable benefit at a delay of at least 12 weeks from diagnosis until definitive surgery.

Three-dimensional (3D) 18F-fluorodeoxyglucose (FDG) positron emission tomography/computed tomography (PET/CT) is obtained routinely for the initial staging of esophageal cancer and has been shown in several sites to alter the gross tumor volume (GTV) delineation [5-8]. Specifically in the esophagus, 3D $\mathrm{PET} / \mathrm{CT}$ has been shown to improve staging [9]. Theoretically, $\mathrm{PET} / \mathrm{CT}$ could eliminate the need for additional staging methods in patients with distant metastatic disease [3]. This would expedite treatment and avoid potentially ineffective treatment methods. Not only does 3D PET/CT identify the primary tumor location, it is an early assessment tool for treatment response, outcome prediction, and therapy modification [10-12]. FDG PET is able to detect most primary tumors and lymph nodes. Studies have demonstrated a sensitivity of $30-93 \%$ and a specificity of 79-100\% for lymph node detection [13].

Endoscopically-placed fiducial markers have facilitated determination of respiratory associated tumor motion in the treatment of esophageal cancer as well as strategies of abdominal compression to decrease such motion, which has enhanced a conformal approach, particularly when used in conjunction with image-guided radiation therapy (IGRT) [14]. When planning esophageal cancer radiotherapy treatment, it is common that accounting for microscopic disease, nodal involvement, and tumor motion is associated with larger planning target volumes (PTV) and consequently, there is increased concern about the amount of normal tissue irradiated [15]. Studies have shown that increased areas of irradiated tissues can result in harmful effects such as radiation pneumonitis, pericardial effusion, and pleural effusion [15]. At our institution, all trimodality esophageal cancer patients receive 3D PET/CT imaging before treatment. In a study on 81 patients with esophageal cancer evaluating the displacement of metal fiducial markers between the digitally reconstructed radiographs and on-board kilovoltage images, respiratory associated superior-inferior tumor movements of $1.25 \mathrm{~cm}$ for proximal and middle esophageal tumors, and $1.75 \mathrm{~cm}$ for those in the distal esophagus were noted [15].

The role of $3 \mathrm{D}$ PET/CT in esophageal tumors that move with respiration and have the potential for significant mucosal inflammation is unclear. The Gastrointestinal $(\mathrm{Gl})$ research group at Moffitt Cancer Center previously reported the stability data for esophageal fiducial markers endoscopically implanted under ultrasound guidance within $1 \mathrm{~cm}$ from the superior and in-
- Table 1 Patient characteristics.

\begin{tabular}{|l|c|}
\hline Characteristic & \\
\hline Age (median), years & 66 \\
\hline Gender, n (\%) & $32(78.0)$ \\
\hline - Male & $8(19.5)$ \\
\hline - Female & \\
\hline Location of tumor, n (\%) & $1(2.4)$ \\
\hline - Upper/middle & $3(7.3)$ \\
\hline - Middle & $2(4.9)$ \\
\hline - Middle/lower & $29(70.7)$ \\
\hline - Lower & $4(9.8)$ \\
\hline - GEJ/lower & $1(2.4)$ \\
\hline - GEJ & 26.0 \\
\hline MTV (median), cm3 & \\
\hline Pathology, n (\%) & $5(12.5)$ \\
\hline - SCC & $35(87.5)$ \\
\hline - Adenocarcinoma & \\
\hline GEJ, gastroesophageal junction; MTV, metabolic tumor volume; SCC, squa- \\
mous cell carcinoma.
\end{tabular}

ferior edges of the tumor [14]. However, the correlation between gross tumor volumes derived from $3 \mathrm{D}$ PET/CT versus endoscopically placed fiducial markers has not yet been reported. This study tested the correlation between metabolic tumor volumes (MTV) derived from 3D PET/CT and endoscopically placed fiducial markers using ultrasonography.

\section{Materials and methods}

\section{Patient population}

In total, 62 patients with esophageal cancer were selected for this retrospective, IRB-approved analysis (MCC 16567 - initially approved 14 July 2011) with waived informed consent. This study was approved by the appropriate ethics committee and has been performed in accordance with the ethical standards laid down in the 1964 Declaration of Helsinki and its later amendments. A total of 22 patients were excluded from the study as they only received one fiducial marker due to obstruction inhibiting passage of the endoscope to the inferior extent of the mass, extension of tumor into the stomach, or other technical difficulties. All patients included in the study underwent placement of a VISICOIL ${ }^{\mathrm{TM}}$ (RadioMed Corporation, an IBA Company, Bartlett, TN, United States) $10 \mathrm{~mm} \times 0.75 \mathrm{~mm}$ gold fiducial marker at the inferior and superior borders of the tumor and received 3D PET/CT before radiotherapy (RTX). Refer to

- Table 1 for patient statistics and characteristics. In total, 20 patients received fiducials pre-PET/CT and 20 patients received fiducials post-PET/CT. In cases where patients underwent fiducial placement post-PET/CT, planning CTs were used to delineate the location of the fiducial marker. The $3 \mathrm{D}$ planning $\mathrm{CTS}$ 
were imported from the Pinnacle treatment planning system (TPS; Version 9.8 Philips Medical System ${ }^{\mathrm{TM}}$, Fitchburg, WI, United States). The 3D PET/CT and planning CT images were imported into an image analysis software system (Mirada RTx, Mirada Medical, Oxford, UK) for measurements.

\section{Measurement of MTV}

Patients were imaged with a GE Discovery STE PET/CT Scanner (GE Medical Systems) at our institution. A tumor threshold was created using a background uptake method ( $\mathbf{F i g . 1}$ ). A $3 \mathrm{~cm}$ spherical volume-of-interest (VOI) was dropped onto a homogenous uptake region in the liver. The mean and standard deviation of the standardized uptake value (SUV) was extracted to calculate a threshold for the tumor volume as shown in Equation 1.

\section{MTV threshold $=\left[\right.$ liver $_{\mu}+2$ liver $\left._{\sigma}\right]$}

where $\mu$ is mean and $\sigma$ is standard deviation. In cases where contours extended into the stomach or the heart, a Boolean tool was used to create a conformal MTV. These difficult contours were then physician-verified and/or edited. The fiducial was delineated on CT via an absolute threshold for Hounsfield Unit (HU) greater than 350. The centroid was determined as the center of mass of the fiducial contour.

\section{Measurement of fiducial distance}

The MTV contour was specified using the above defined liver threshold method and the axial slices were used to measure the distance between each centroid and the corresponding tumor border. This distance was defined as the number of slices between the centroid of the fiducial and the first axial PET slice that included the MTV contour ( $\triangleright$ Fig. 2). The number of axial slices was then multiplied by slice thickness (3.27 mm for PET/ CT and $3.0 \mathrm{~mm}$ for planning $\mathrm{CT}$ ) to provide the distance in centimeters. The distance and absolute values of the distances were recorded. Negative values described distances where fiducials were located inferior to the MTV border for both the superior and inferior margins of the tumor. Descriptive statistics such as: mean, median, standard deviation, maximum, and minimum were calculated. Cases involving large distances were investigated. The Concordance Correlation Coefficient (CCC) was used to determine the correlation between the MTV threshold and superior fiducial distance (SFD), MTV threshold and inferior fiducial distance (IFD), tumor site and IFD, tumor site and SFD, time between fiducial and $P E T / C T$, patient age and IFD, and patient age and SFD. Tumor site represents the location of the tumor in the esophagus (upper, mid or distal/gastroesophageal junction (GEJ)). The strength-of-agreement scale was as follows: CCC >0.99: high; CCC 0.95-0.99: substantial; CCC 0.90 - 0.95: moderate; CCC <0.90: poor [16].

\section{Image feature extraction and analysis}

An in-house program extracted image features from each MTV contour. The details of this feature extraction are provided in Oliver et al. [17]. In total, 81 image features were extracted including 11 shape features, 22 intensity features, 26 Gray Level Co-occurrence Matrix (GLCM) features, 11 Run Length Matrix

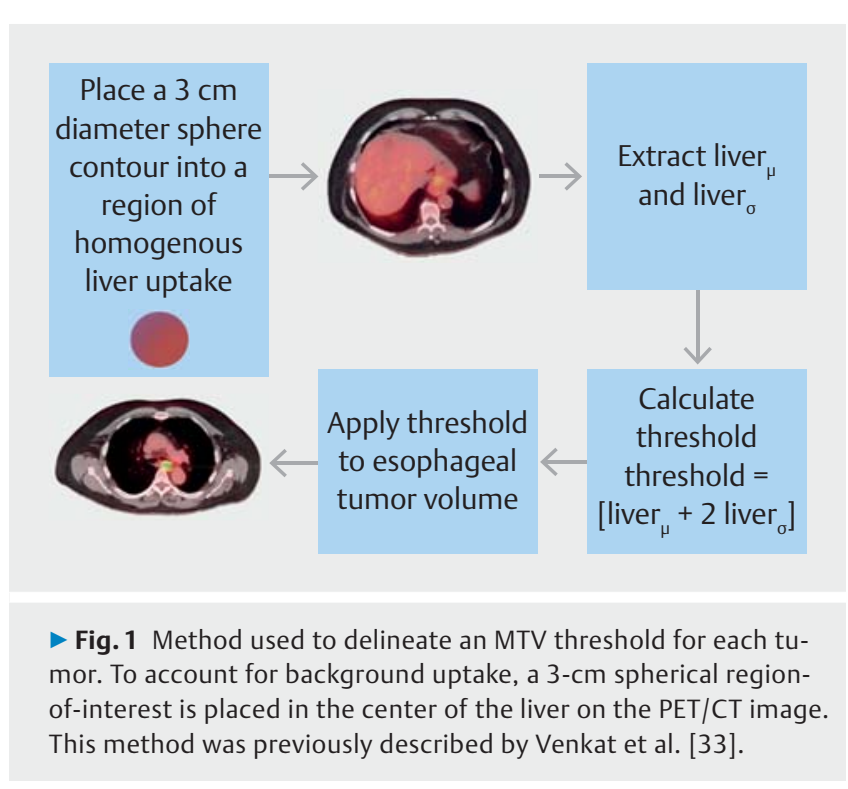

(RLM) features and 11 Gray Level Size Zone Matrix (GLSZM) features [18-21]. Co-occurrence matrices were extracted with dimensions of $128 \times 128$ and were calculated based on the $3 \mathrm{D}$ images with a step size of 1 voxel in 13 directions. Gray levels were binned into 128 levels with equal intensity intervals for the run-length matrices. The run length was calculated with the $3 \mathrm{D}$ images in 13 directions (defined by Xu et al. [22,23]).

\section{Statistical analysis}

Of the patient cohort, 17 patients had pathological response data. For these cases, an independent samples $t$ test was performed comparing the mean consistency scores of two patient groups: pathological complete responder (PCR) to treatment versus pathological partial responder (PPR).

\section{Results}

The median MTV threshold was 2.50 SUV (range: 1.6 - 3.6) with relative uptake of $23 \%$ (range: $5-79 \%$ ) for all patients. For patients receiving fiducials before undergoing PET/CT (PrePF), the median MTV threshold was 2.45 SUV (1.6-3.6). For patients receiving fiducials after undergoing PET/CT (PostPF), the median MTV threshold was 2.6 SUV $(1.8-3.4)$ ( Table 2, > Table 3, - Table4). The median distance between MTV and fiducials was $-0.6 \mathrm{~cm}(-3.90 \mathrm{~cm}$ to $2.70 \mathrm{~cm})$ and $1.16 \mathrm{~cm}(-4.2 \mathrm{~cm}$ to $6.87 \mathrm{~cm}$ ) for inferior and superior tumor borders, respectively. PrePF patients ( $>$ Table 3 ) demonstrated a median distance between MTV and fiducials of $-0.82 \mathrm{~cm}(-3.60 \mathrm{~cm}$ to $2.62 \mathrm{~cm})$ and $1.64 \mathrm{~cm}(-0.33 \mathrm{~cm}$ to $6.87 \mathrm{~cm})$ for inferior and superior borders, respectively. PostPF patients ( $>$ Table 4 ) demonstrated a median distance between MTV and fiducials of $0.30 \mathrm{~cm}$ $(-3.90 \mathrm{~cm}$ to $2.70 \mathrm{~cm})$ inferiorly and $0.75 \mathrm{~cm}(-4.20 \mathrm{~cm}$ to $4.20 \mathrm{~cm}$ ) superiorly. A poor strength-of-agreement (CCC $<0.90$ ) was calculated between MTV threshold and superior fiducial distance (SFD), MTV threshold and inferior fiducial distance (IFD), tumor site and IFD, tumor site and SFD, time 


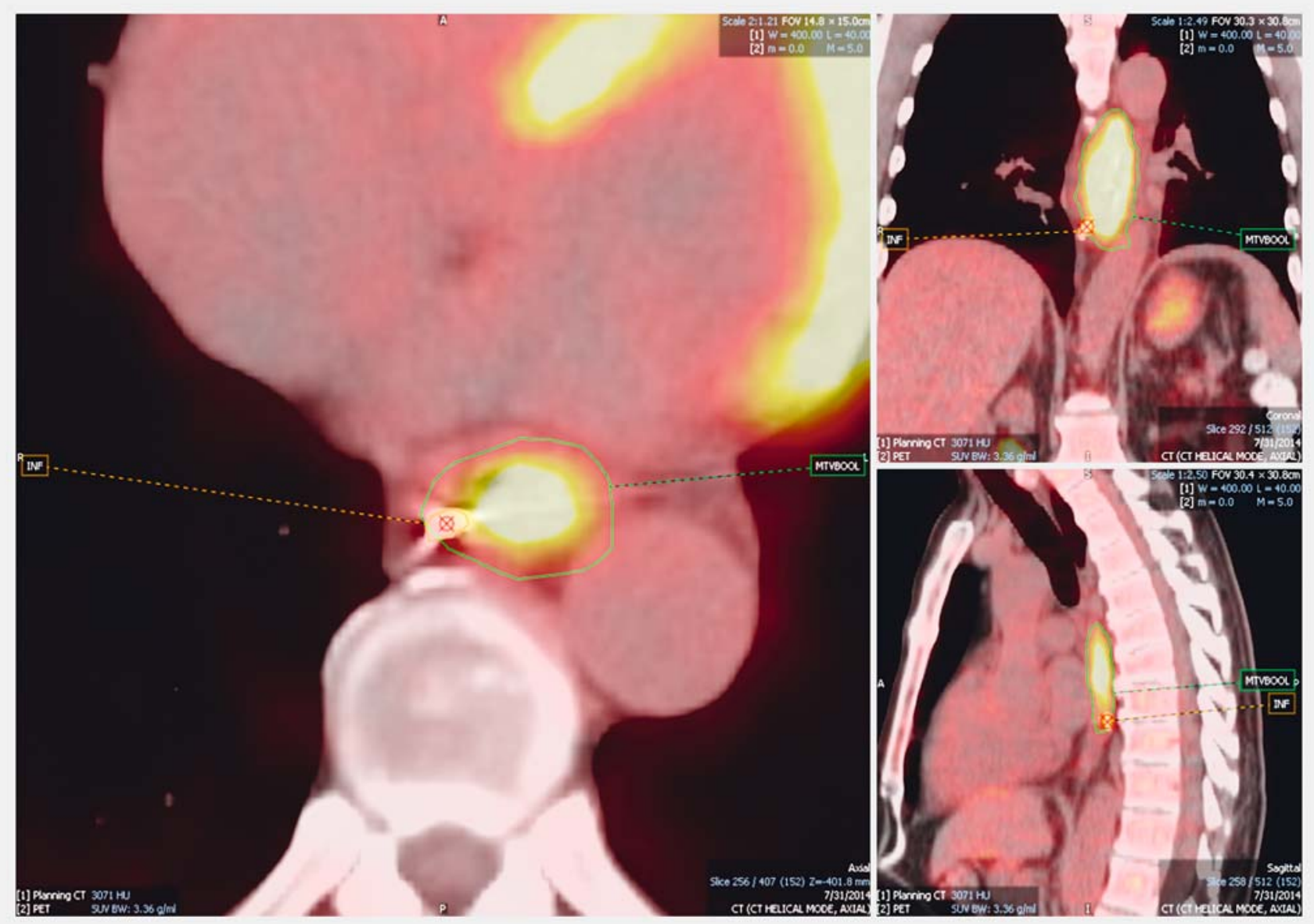

- Fig. 2 Method of identifying the fiducial and marking the centroid at the inferior border of the MTV.

- Table 2 Fiducials vs MTV border.

\begin{tabular}{|l|c|c|c|c|c|}
\hline All patients & Inferior fiducial distance, $\mathbf{c m}$ & Superior fiducial distance, $\mathbf{c m}$ & MTV threshold, SUV & MTV threshold, $\%$ & MTV, $\mathbf{c m}^{\mathbf{3}}$ \\
\hline Mean & -0.42 & 1.16 & 2.51 & 29 & 30.91 \\
\hline Median & -0.60 & 1.25 & 2.50 & 23 & 22.40 \\
\hline Min & -3.90 & -4.20 & 1.60 & 5 & 7.80 \\
\hline Max & 2.70 & 6.87 & 3.60 & 79 & 18 \\
\hline SD & 1.50 & 1.70 & 0.43 & 28.04 \\
\hline MTV, metabolic tumor volume; SUV, standardized uptake value.
\end{tabular}

between fiducial and PET/CT, patient age and IFD, and patient age and SFD.

In total, 22 of $40(55.0 \%)$ patients had inferior fiducials located superior to the MTV border ( $\triangleright$ Fig. $3 a$ ). In 14 patients (35.0\%), inferior fiducials were below the MTV border. Four patients $(10.0 \%)$ demonstrated perfect agreement between the inferior fiducial and MTV border (all PrePF). The superior fiducial and MTV border did not have perfect agreement in any case. In 33 patients (82.5\%), the superior fiducial was located inferior to the MTV border ( $\triangleright$ Fig. 3b). In 7 of 40 patients (17.5\%), the superior fiducial was located superior to the MTV border. In 9 of 40 (22.5\%) patients, distances to the MTV border were less than $0.5 \mathrm{~cm}$ for the inferior fiducial, and in 3 of $40(7.5 \%)$ patients, distances to the MTV border were less than $0.5 \mathrm{~cm}$ for the superior fiducial (> Fig. 3 ).

The data were analyzed excluding five patients in whom the interval between fiducial placement and PET/CT was greater than 30 days. No significant differences in the results were detected without these five patients in the analysis. 
- Table 3 PrePF fiducials vs MTV border.

\begin{tabular}{|l|c|c|c|c|c|}
\hline PrePF & Inferior fiducial distance, $\mathbf{c m}$ & Superior fiducial distance, $\mathbf{c m}$ & MTV threshold, SUV & MTV threshold, $\%$ & MTV, $\mathbf{c m}^{\mathbf{3}}$ \\
\hline Mean & -0.70 & 1.64 & 2.44 & 24 & 40.13 \\
\hline Median & -0.82 & 1.64 & 2.45 & 19 & 34.70 \\
\hline Min & -3.60 & -0.33 & 1.60 & 5 & 3.00 \\
\hline Max & 2.62 & 6.87 & 3.60 & 74 & 107.1 \\
\hline SD & 1.42 & 1.44 & 0.42 & 16 & 33.47 \\
\hline
\end{tabular}

PrePF, patients receiving fiducials before undergoing PET/CT; MTV, metabolic tumor volume; SUV, standardized uptake value.

- Table4 PostPF fiducials vs MTV border.

\begin{tabular}{|l|c|c|c|c|c|}
\hline PostPF & Inferior fiducial distance, $\mathbf{c m}$ & Superior fiducial distance, $\mathbf{c m}$ & MTV threshold, SUV & MTV threshold, \% & MTV, $\mathbf{c m}^{\mathbf{3}}$ \\
\hline Mean & -0.14 & 0.69 & 2.58 & 35 & 22.15 \\
\hline Median & -0.30 & 0.75 & 2.60 & 31 & 20.60 \\
\hline Min & -3.90 & -4.20 & 1.80 & 11 & 7.80 \\
\hline Max & 2.70 & 4.20 & 3.40 & 79 & 19 \\
\hline SD & 1.74 & 1.85 & 0.43 & 74.80 \\
\hline
\end{tabular}

PostPF, patients receiving fiducials after undergoing PET/CT; MTV, metabolic tumor volume; SUV, standardized uptake value.

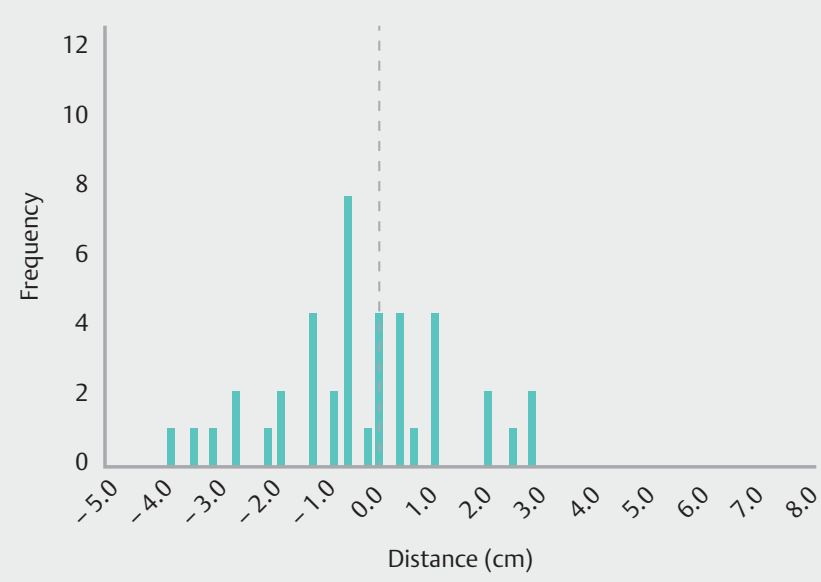

a $\quad *$ Negative values denote that fiducials were located inside MTV.

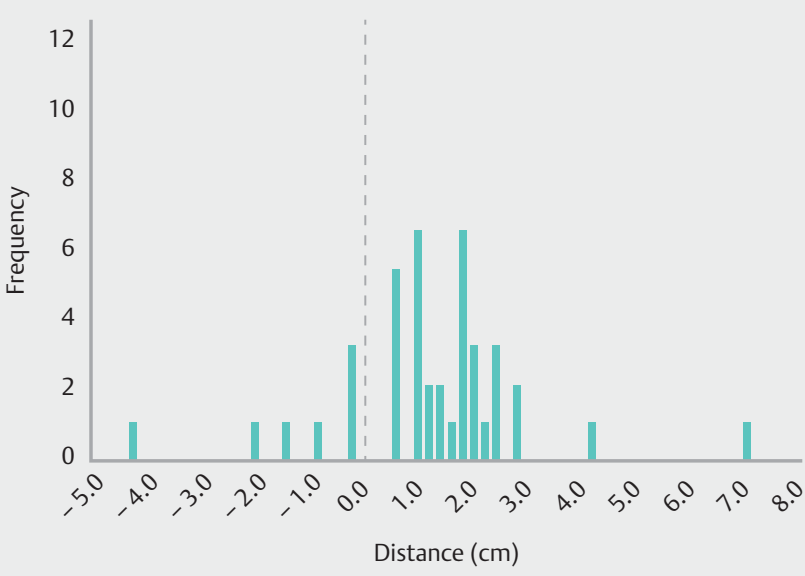

b $\quad *$ Negative values denote that fiducials were located outside MTV.

- Fig. 3 Inferior discordance (a) and superior discordance (b) histograms. Note: For inferior discordance, negative values denote that fiducials were located inside MTV. For superior discordance, negative values denote that fiducials were located outside MTV.

\section{Large discordances}

In 10 patients, the superior fiducial-MTV discordance was greater than $2.0 \mathrm{~cm}$. Of these patients, the time between fiducial placement and PET ranged from 2 to 141 days. There were only two occurrences where the superior fiducial was inferior to the MTV border (negative distance). In the case of the patient with $6.87 \mathrm{~cm}$ discord (maximum discordance, see $>$ Fig. 4), the patient was diagnosed with extensive esophagitis and several nodules at the gastroesophageal junction (GEJ). This discord was attributed to esophagitis. One of the occurrences where the superior fiducial was superior to the MTV border, was in the only patient with stage 1 cancer (medically inoperable). The patient was diagnosed with adenocarcinoma of the distal esophagus although the uptake was in the mid esophagus. This patient had Barrett's esophagus from the mid esophagus to the GEJ which may have influenced the uptake in the mid esophageal region ( $\triangleright$ Fig.5). There was no correlation between MTV-to-fiducial distances greater than $2 \mathrm{~cm}$ and the gastroenterologist who performed the fiducial implantation. 


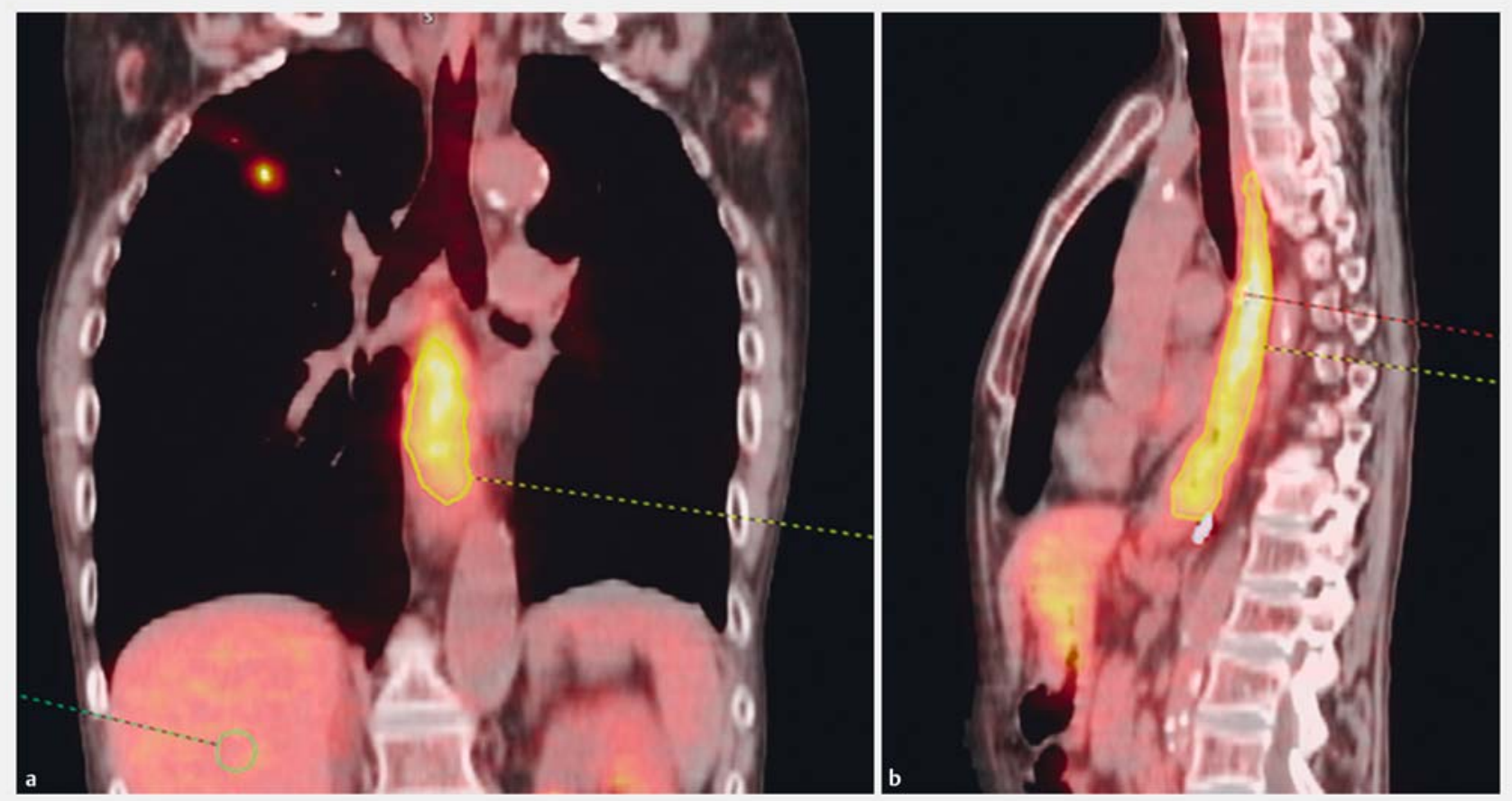

- Fig. 4 Patient with largest discordance $(6.87 \mathrm{~cm})$ between superior fiducial and superior MTV border. a Coronal view. b Sagittal view.
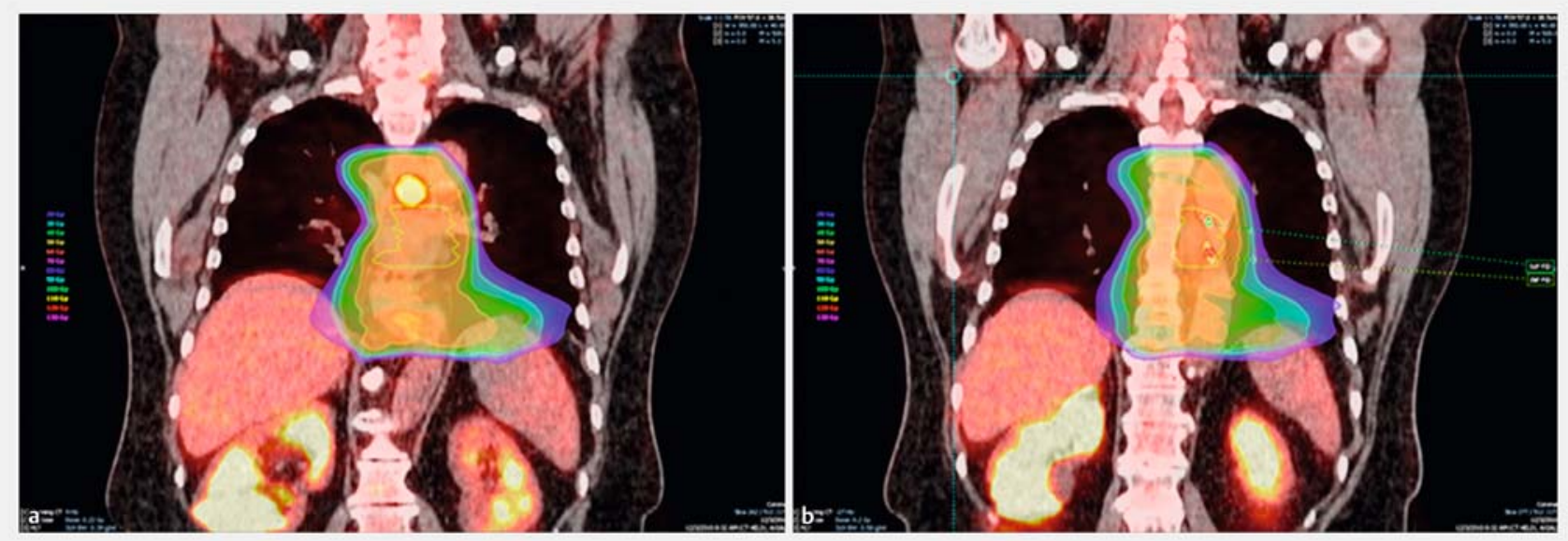

- Fig. 5 Example of discordance between fiducials and MTV at the inferior and superior location $(-4.20 \mathrm{~cm}$ discordance between superior fiducial and superior MTV border). a An image of the MTV location (red contour). b An image of the superior fiducial (green dot) and inferior fiducial (red dot) location. The GTV (yellow contour) and isodose lines are also shown (orange, green, blue, and purple regions) in (a) and (b).

\section{Radiomic feature results}

The radiomic feature, eccentricity, was the only feature that demonstrated statistically significant associations with pathologic response. It is a measure of the non-circularity of the tumor and is defined by the ratio of the minor tumor axis to the major tumor axis where a value of 0 represents a perfect circle and 1 represents a line [24]. The result from independent $t$ tests indicated that $P C R$ patients (mean $=0.333, S D=0.112, N=10$ ) scored a higher value of radiomic feature eccentricity than the PPR patient group (mean $=0.217, S D=0.0817, N=7, t(15)=$
2.327, $P<0.034$, two-tailed). In addition, the assumption of homogeneity of variances was tested and satisfied via Lavene's $F$ test, $F(15)=0.715, P=0.411$. The difference of the scale points $(d=1.196)$ was found to exceed Cohen's conventions for large effect $(d=0.8)$ [25] and the 95\% confidence interval around the difference between the group means was relatively precise (0.0098-0.222). Spearman's rho correlation coefficients revealed a statistically significant trend $(r=-0.056, P=0.024)$ between the value of radiomic feature eccentricity and pathological response ( Fig. $\mathbf{6})$. 


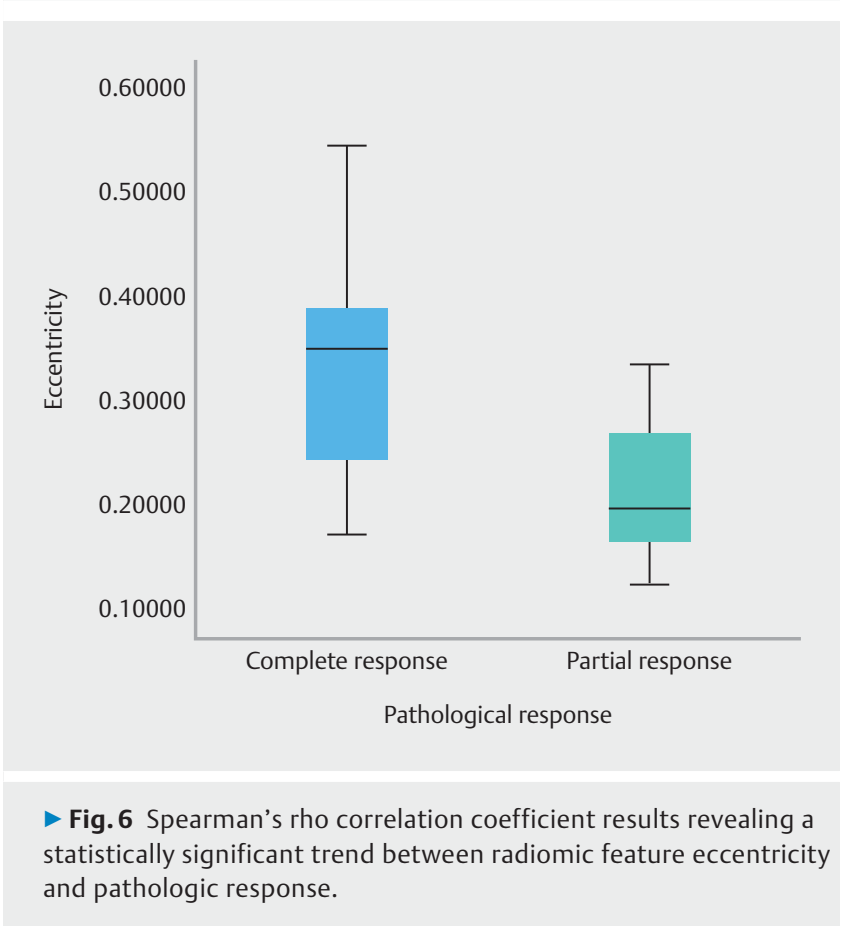

\section{Discussion}

Esophageal tumors can have significant respiration-induced tumor motion. A study by jin et al. measured the peak-to-peak magnitudes of the motion [26]. The greatest motion was found for the distal esophagus in the cranial-caudal direction with a median distance of $5.4 \mathrm{~mm}$. Median displacements for the proximal and middle esophagus were $2.9 \mathrm{~mm}$ and $3.7 \mathrm{~mm}$, respectively. Interestingly, motion in the cranial-caudal direction was shown to have the strongest correlation with respiratory curves [27]. In that particular study, motion in the cranialcaudal direction maximally reached $13.8 \mathrm{~mm}$ in the lower thoracic esophagus, $7.4 \mathrm{~mm}$ in the middle esophagus, and $4.3 \mathrm{~mm}$ in the upper esophagus. Investigators are beginning to realize the benefit of fiducials in radiation treatment planning for cancers of the esophagus [28] and studies have demonstrated that implantation of esophageal fiducial markers are both safe and feasible for target volume delineation purposes on CT [28]. However, to our knowledge, the discordance between endoscopically placed fiducial markers and PET MTV in esophageal cancer has not been investigated.

A retrospective study of esophageal cancer patients treated with preoperative or definitive chemoradiation at our institution between 2000 and 2012 demonstrated 3-year overall survival rates of $44.8 \%$ for $3 \mathrm{D}$ conformal radiation therapy (3DCRT) and $41.5 \%$ for intensity-modulated radiation therapy (IMRT) [29]. The rates for that study agree well with the national 5-year relative survival rate of $17 \%$ between 2010 and 2012 [30 - 32]. In the current study, patients were treated with radiotherapy between 2009 and 2014.

At our institution, it is common practice for patients with esophageal tumors that move with respiration to undergo fiducial placement in addition to 18F-FDG PET/CT to facilitate conformal delivery of a simultaneous integrated boost to the gross tumor volume [33]. Our data recently reported a $56 \%$ complete pathologic response rate [34], significantly higher than the 29 \% reported with the current standard of care CROSS regimen [35], which may be secondary to integration of both fiducials and MTV. Indeed, it is perhaps the combination of fiducials and MTV that may allow for precise dose painting - the treatment of tumor areas that are more metabolically active with higher doses of radiation. This incorporation has facilitated our confidence in dose painting gross disease to a total dose of 56 Gy in 28 fractions while simultaneously treating areas at risk of microscopic spread of disease to 50.4 Gy while ensuring the reproducibility of our daily image guided delivery. Focal dose escalation is of particular concern in the region of the gastroesophageal junction where stomach filling can cause additional motion [36]. The fiducials delimit the visible endoscopic mucosal tumor burden which improves target volume delineation in precise conjunction with daily, image guided dose delivery. MTV, on the other hand, identifies metabolically active tumor regions and submucosal microscopic spread of disease that may not be visible endoscopically or on a CT image.

Although our hypothesis in this study was neither confirmed nor disproven, a strong correlation was found between the inferior fiducial location and the border of the MTV, and the absolute SUV values defining the MTV were reasonably close to commonly used values of 2.5. However, relative SUV values were lower than the typical values of $40 \%$ of SUVmax. A study by Zhong et al. found that an SUV cutoff of 2.5 was best to estimate gross tumor length in squamous cell carcinoma of the esophagus using 18F-FDG PET [37]. This agrees with our median MTV threshold of 2.51 SUV.

Some patients demonstrated large areas of uptake that extended well beyond the implanted fiducial $(-4.2$ to $6.9 \mathrm{~cm})$ as seen in the patient diagnosed with esophagitis as discussed above, while in other instances, tumors had small MTVs with large distances between the MTV border and fiducial location. These could represent tumors that were not FDG avid. The results demonstrated that, in $82.5 \%$ of patients, the superior fiducial was located below the MTV threshold. Thus, in these patients, the MTV extended above the superior fiducial placement. This discordance could be due to inflammation or esophagitis or potentially to disease that was not endoscopically visible. In comparison, the MTV extended below the inferior fiducial in only $55 \%$ of patients. Of these patients, 5 of 33 (15.2\%) had fiducial/MTV border distances less than $0.7 \mathrm{~cm}$ at the superior tumor border and 8 of 22 (36.4\%) had fiducial/MTV border distances less than $0.7 \mathrm{~cm}$ at the inferior tumor border. The majority of these patients were diagnosed as distal or GEJ tumors. Thus, accurate fiducial placement may not have been possible in these patients given the proximity of the stomach or disease extension into the stomach. There was a clear indication that, in most patients, the MTV extended beyond the superior fiducial and that timing of the PET before or after fiducial placement was not a significant factor.

The etiology of the discordance superiorly is unclear, however, with PET/CT showing high uptake at and above the endoscopically placed marker, potentially representing benign secondary esophagitis such as in the setting of luminal obstruc- 
tion, the presence of malignant nodes, inflammation caused by the technical aspects of the fiducial placement itself, or potential submucosal disease. In the case of the largest discordance, the patient had been diagnosed with extensive esophagitis before treatment. The inferior fiducial and inferior MTV border are well correlated in our cohort. This supports the idea that the inferior fiducial marker is a good surrogate for inferior tumor extent. With the use of daily image guidance, where we can set up to the inferior fiducial, we may be able to limit our inferior CTV to PTV margins, which accounts for patient set-up uncertainty. This is particularly true for GEJ tumors, where stomach filling has been shown to distort coverage of boost volumes when simultaneous integrated boost IMRT planning is used [36].

Ideally, we would perform pathologic assessment to evaluate which (fiducial location or MTV) is more accurate in delineating gross disease. However, this was a retrospective study and tissue was unavailable. Also, there is a high pathologic response rate in this cohort, which would make accurate posttreatment pathologic assessment impossible. Given this limitation of our study, we are not suggesting that fiducial location is superior to $3 \mathrm{D} P E T / C T$ in delineating the GTV or vice versa. Instead, we posit that both fiducial placement and PET/CT should be performed when possible and should be interpreted in conjunction with each other. If they correlate well, perhaps we can more confidently use a smaller PTV margin. If they are discordant, we can adjust our PTV margins accordingly. Furthermore, when an inferior fiducial marker is unable to be placed due to obstruction, tumor extent into the stomach or other technical difficulties, the MTV can be used as an acceptable surrogate for fiducial placement inferiorly during target delineation, but additional PTV margin should be used due to less accurate daily patient set-up. The discordance superiorly suggests caution should be used when modifying the PTV margin superiorly.

One limitation of this study is that half of the patients underwent PET/CT imaging before fiducial implantation. Thus, the PET/CT and planning CT had to be fused, and the fiducials and distances between fiducials and the MTV borders had to be identified and measured. Image fusion may have led to some uncertainty [38].

In this study, the Radiomic feature eccentricity demonstrated predictability between PCR and PPR patients where a higher eccentricity value corresponded with PCR patients ( $\triangleright$ Fig.6). Thus, PPR patients were associated with more circular tumors in comparison to PCR patients. A study by O'Sullivan et al. demonstrated that eccentricity was a strong prognostic indicator for time to death (survival) in sarcoma patients [39]. Our study is limited because of the number of patients, however, and a larger study is warranted to uncover clinical relevance. A similar study which evaluated textural features for prediction of therapy response in esophageal cancer, demonstrated that textural features were more efficient than SUV in identifying complete responders [2].

\section{Conclusion}

The inferior fiducial location and MTV border for esophageal cancer had a strong correlation in comparison to the superior MTV border and corresponding fiducial. Given the concordance inferiorly and the ability to more precisely set up the patient with daily image guidance matching to fiducials, it may be possible to minimize the PTV margin inferiorly in select patients, thereby, limiting dose to normal structures, especially in patients with focal dose escalation in tumors involving the GEJ. The etiology of the discordance between the superior fiducial location and MTV border could be caused by inflammation from the fiducial placement itself, submucosal disease, or benign secondary esophagitis. Regardless of the discordance, having both fiducials is important for image guidance. Further study is needed to determine the factors confounding FDG uptake superiorly to optimize MTV delineation and target volumes.

\section{Acknowledgments}

J. Oliver received funding from the Florida Education Fund McKnight Doctoral Fellowship.

Competing interests

None

References

[1] American Cancer Society. Cancer facts \& figures 2015. Atlanta: 2015

[2] Tixier F, Le Rest CC, Hatt M et al. Intratumor heterogeneity characterized by textural features on baseline 18 F-FDG PET images predicts response to concomitant radiochemotherapy in esophageal cancer. J Nucl Med 2011; 52: 369-378

[3] Shridhar R, Almhanna K, Meredith KL et al. Radiation therapy and esophageal cancer. Cancer Control 2013; 20: $97-110$

[4] Dittrick GW, Weber JM, Shridhar R et al. Pathologic nonresponders after neoadjuvant chemoradiation for esophageal cancer demonstrate no survival benefit compared with patients treated with primary esophagectomy. Ann Surg Oncol 2012; 19: 1678-1684

[5] Spratt DE, Diaz R, McElmurray J et al. Impact of FDG PET/CT on delineation of the gross tumor volume for radiation planning in nonsmall-cell lung cancer. Clin Nucl Med 2010; 35: 237-243

[6] Zheng $Y$, Sun $X$, Wang J et al. FDG-PET/CT imaging for tumor staging and definition of tumor volumes in radiation treatment planning in non-small cell lung cancer. Oncol Lett 2014; 7: 1015-1020

[7] Gregoire V, Haustermans K, Geets X et al. PET-based treatment planning in radiotherapy: a new standard? J Nucl Med 2007; 48: (Suppl. 01): $68 \mathrm{~S}-77 \mathrm{~S}$

[8] Grégoire V, Haustermans K, Lee J. Chapter 20. Molecular imageguided radiotherapy with positron emission tomography; 20.3 PET image segmentation. In: Joiner MC, van der Kogel A, eds. Basic Clinical Radiobiology. 4th edn. CRC Press; 2009: 271-286

[9] Wang YC, Hsieh TC, Yu CY et al. The clinical application of 4D 18F-FDG $\mathrm{PET} / \mathrm{CT}$ on gross tumor volume delineation for radiotherapy planning in esophageal squamous cell cancer. J Radiat Res 2012; 53: 594-600 
[10] Westerterp M, Westreenen HLv, Reitsma JB et al. Esophageal cancer: $\mathrm{CT}$, endoscopic US, and FDG PET for assessment of response to neoadjuvant therapy - systematic review. Radiology 2005; 236: $841-851$

[11] Wieder HA, Ott K, Lordick F et al. Prediction of tumor response by FDG-PET: comparison of the accuracy of single and sequential studies in patients with adenocarcinomas of the esophagogastric junction. Eur J Nucl Med Mol Imaging 2007; 34: 1925 - 1932

[12] Lordick F, Ott K, Krause B] et al. PET to assess early metabolic response and to guide treatment of adenocarcinoma of the oesophagogastric junction: the MUNICON phase II trial. Lancet Oncol 2007; 8: 797-805

[13] Muijs CT, Beukema JC, Pruim J et al. A systematic review on the role of FDG-PET/CT in tumour delineation and radiotherapy planning in patients with esophageal cancer. Radiother Oncol 2010; 97: 165-171

[14] Fernandez DC, Hoffe SE, Barthel JS et al. Stability of endoscopic ultrasound-guided fiducial marker placement for esophageal cancer target delineation and image-guided radiation therapy. Pract Radiat Oncol 2013; 3: $32-39$

[15] Fukada J, Hanada T, Kawaguchi O et al. Detection of esophageal fiducial marker displacement during radiation therapy with a 2-dimensional on-board imager: analysis of internal margin for esophageal cancer. Int J Radiat Oncol Biol Phys 2013; 85: 991 - 998

[16] McBride GB. Equivalence measures for comparing the performance of alternative methods for the analysis of water quality variables. Auckland, New Zealand: National Institute of Water \& Atmospheric Research Ltd; 2007

[17] Oliver JA, Budzevich M, Zhang GG et al. Variability of image features computed from conventional and respiratory-gated PET/CT images of lung cancer. Transl Oncol 2015; 8: 524-534

[18] Haralick RM, Shanmugam K, Dinstein IH. Textural features for image classification. IEEE Trans Systems Man Cybernet 1973: SMC-3 610621

[19] Galloway MM. Texture analysis using gray level run lengths. Comput Graph Image Process 1975; 4: 172-179

[20] Tustison NJ, Gee JC. Runlength matrices for texture analysis. Insight J; 2008: http://hdl.handle.net/1926/1374

[21] Thibault G, Fertil B, Navarro C et al. Texture indexes and gray level size zone matrix. Application to cell nuclei classification. Pattern Recognit Inf Process 2009: 140 - 145

[22] Xu D-H, Kurani AS, Furst JD, Raicu DS. Run-length encoding for volumetric texture. The 4th IASTED International Conference on Visualization, Imaging and Image Processing; VIIP. Marbella, Spain: 2004

[23] Kurani AS, Xu D-H, Furst JD, Raicu DS. Co-occurrence matrices for volumetric data. Paper presented at: The 7th IASTED International Conference on Computer Graphics and Imaging; CGIM. August 1719, Kauai, Hawaii, United States: 2004

[24] El Naqa I, Grigsby P, Apte A et al. Exploring feature-based approaches in PET images for predicting cancer treatment outcomes. Pattern Recognit 2009; 42: $1162-1171$
[25] Murphy KR, Myors B. Statistical power analysis: A simple and general model for traditional and modern hypothesis tests. 2nd edn. Mahwah, NJ: Lawrence Erlbaum Associates; 2004

[26] Jin P, Hulshof MC, de Jong R et al. Quantification of respiration-induced esophageal tumor motion using fiducial markers and fourdimensional computed tomography. Radiother Oncol 2016; 118: $492-497$

[27] Yamashita H, Kida S, Sakumi A et al. Four-dimensional measurement of the displacement of internal fiducial markers during 320-multislice computed tomography scanning of thoracic esophageal cancer. Int J Radiat Oncol Biol Phys 2011; 79: 588-595

[28] Machiels M, Van Hooft J, Jin P et al. Endoscopy/EUS-guided fiducial marker placement in patients with esophageal cancer: a comparative analysis of 3 types of markers. Gastrointest Endosc 2015; 82: 641 649

[29] Freilich J, Hoffe SE, Almhanna K et al. Comparative outcomes for three-dimensional conformal versus intensity-modulated radiation therapy for esophageal cancer. Dis Esophagus 2015; 28: 352 - 357

[30] American Cancer Society. Cancer facts \& figures 2010. Atlanta: 2010

[31] American Cancer Society. Cancer facts \& figures 2011. Atlanta: 2011

[32] American Cancer Society. Cancer facts \& figures 2012. Atlanta: 2012

[33] Venkat P, Oliver JA, Jin W et al. Prognostic value of ${ }^{18} \mathrm{~F}-\mathrm{FDG}$ PET/CT metabolic tumor volume for complete pathologic response and clinical outcomes after neoadjuvant chemoradiation therapy for locally advanced esophageal cancer. J Nucl Med Radiat Ther 2016; 7: 1-8

[34] Venkat P, Shridhar R, Naghavi AO et al. Pathologic complete response and dose-escalation with preoperative dose painting IMRT chemoradiation in esophageal cancer. Int J Radiat Oncol Biol Phys 2015; 93 : S219-S220

[35] Shapiro J, van Lanschot J], Hulshof MC et al. Neoadjuvant chemoradiotherapy plus surgery versus surgery alone for oesophageal or junctional cancer (CROSS): long-term results of a randomised controlled trial. Lancet Oncol 2015; 16: 1090-1098

[36] Bouchard M, McAleer MF, Starkschall G. Impact of gastric filling on radiation dose delivered to gastroesophageal junction tumors. Int J Radiat Oncol Biol Phys 2010; 77: 292 - 300

[37] Zhong X, Yu J, Zhang B et al. Using 18F-fluorodeoxyglucose positron emission tomography to estimate the length of gross tumor in patients with squamous cell carcinoma of the esophagus. Int J Radiat Oncol Biol Phys 2009; 73: 136-141

[38] Latifi K, Zhang G, Stawicki M et al. Validation of three deformable image registration algorithms for the thorax. J Appl Clin Med Phys 2013; 14: 3834

[39] O'Sullivan F, Roy S, O'Sullivan J et al. Incorporation of tumor shape into an assessment of spatial heterogeneity for human sarcomas imaged with FDG-PET. Biostatistics 2005; 6: 293-301 